\title{
ARID1A-mutant and deficient bladder cancer is sensitive to EZH2 pharmacologic inhibition
}

James E. Ferguson III ${ }^{1,2, *, \#,}$, Hasibur Rehman ${ }^{1 *}$, Darshan S. Chandrashekar ${ }^{3 *}$, Balabhadrapatruni V. S. K. Chakravarthi ${ }^{3}$, Saroj Nepal ${ }^{3}$, Marie-Lisa Eich ${ }^{7}$, Alyncia D. Robinson $^{3}$, Sumit Agarwal ${ }^{3}$, Sai Akshaya Hodigere Balasubramanya ${ }^{3}$, Gurudatta Naik ${ }^{2}$, Upender Manne ${ }^{2,3}$, George $\mathrm{J} \mathrm{Netto}^{3}$, Chong-xian Pan ${ }^{5}$, Guru Sonpavde ${ }^{6}$, Sooryanarayana Varambally $2,3,4, \#$

${ }^{1}$ Department of Urology, The University of Alabama at Birmingham, Birmingham, AL, USA

${ }^{2} \mathrm{O}^{\prime}$ Neal Comprehensive Cancer Center, The University of Alabama at Birmingham, Birmingham, AL, USA

${ }^{3}$ Department of Pathology, The University of Alabama at Birmingham, Birmingham, AL, USA

${ }^{4}$ Informatics Institute, The University of Alabama at Birmingham, Birmingham, AL, USA

${ }^{5}$ Department of Medicine, Brigham and Women's Hospital, Harvard Medical School, Boston, MA, USA

${ }^{6}$ Department of Medicine, Dana-Farber Cancer Institute, Harvard Medical School Boston, MA, USA

${ }^{7}$ Institute of Pathology, University Hospital Cologne, Cologne, Germany.

* Shared primary Authors

\#Senior and corresponding Authors

Correspondence to:

James Ferguson, III M.D., PhD

Department of Urology

O’Neal Comprehensive Cancer Center

Room \# 410D

University of Alabama at Birmingham,

Birmingham, AL 35233, USA

jferguson@uabmc.edu

Sooryanarayana Varambally, Ph.D., Molecular and Cellular Pathology, Department of Pathology,

Wallace Tumor Institute, Room \# 430,

University of Alabama at Birmingham,

Birmingham, AL 35233, USA

Phone: (205) 996-1653

Email: svarambally@uabmc.edu

Running Title: ARID1A deficient bladder cancer is sensitive to EZH2 pharmacologic inhibition. 


\section{Disclosure of Potential Conflicts of Interest:}

Guru Sonpavde, MD was a consultant for BMS, Exelixis, Bayer, Sanofi, Pfizer, Novartis, Eisai, Janssen, Amgen, AstraZeneca, Merck, Genentech, Astellas/Agensys; Research support to institution from Bayer, Amgen, Boehringer-Ingelheim, Merck, Sanofi, Pfizer; Author for Up-todate; Speaker for Clinical Care Options, Physicians Education Resource (PER), Research to Practice (RTP), Onclive 


\begin{abstract}
Metastatic urothelial carcinoma of the bladder is generally incurable by current systemic therapy. Molecular characterization of bladder cancer (BLCa) has revealed multiple candidate driver genes for BLCa tumorigenesis. Epigenetic/chromatin modifiers have been shown to be frequently mutated in BLCa, with ARID1A mutations highly prevalent in nearly $20 \%$ of early and late stage tumors. EZH2 is a histone methyltransferase that acts as an oncogene. The data herein show that ARID1A deficient tumors, but not ARID1A wild-type tumors are sensitive to EZH2 inhibition. Specifically, EZH2 inhibitor-treated ARID1A deficient bladder cancer cells show significantly reduced cell viability, colony formation, and in vivo tumor growth relative to ARID1A-wild type bladder cancer cells. Thus, our study suggests that a specific subset of bladder cancer patients with ARID1A mutations can be therapeutically treated with pharmacologic inhibitors targeting EZH2.
\end{abstract}




\section{Introduction}

Bladder cancer (BLCa) is the 6th most common cancer in the US, and leads to $\sim 18,000$ deaths annually (1). Unfortunately, bladder cancer outcomes have been relatively stagnant despite the recent introduction of a number of new therapies including immunotherapy. Next generation sequencing has revolutionized our understanding of bladder cancer and provides an opportunity to develop personalized therapy $(2,3)$. These analyses have revealed that epigenetic modifying genes are frequently mutated in bladder cancer as over $90 \%$ of tumors harbor inactivating mutations in at least one chromatin modifying enzyme (4). About $20 \%$ of BLCa have truncating and inactivating mutations in the AT Rich Interactive Domain 1A (ARID1A) gene, a member of the SWI/SNF chromatin modifying complex (aka BAF in mammals), making it the most frequently mutated epigenetic gene in bladder cancer. The development of such epigenetic mutations is one of the early events in BLCa tumorigenesis (5).

ARID1A is the DNA-binding component of the large 1.15 MDa SWI/SNF complex. This complex contains ATPase activity which is important for ATP-dependent chromatin remodeling that generally results in increased transcriptional accessibility and modulates diverse gene programs and cellular processes including DNA repair, telomere cohesion, and immune recognition (reviewed in refs $(6,7)$ ). Thus, the functional ramifications of ARID1A deficiency are dependent on its downstream transcriptional consequences, which can be altered by other epigenetic transcriptional regulators and the specific cellular context.

Previously, we and others have shown that the histone methyltransferase Enhancer of Zeste Homolog 2 (EZH2), which generates a transcriptionally repressive chromatin mark, is over-expressed in many aggressive cancers where it is thought to drive growth and is thus considered an oncogene (8-11). EZH2 functions as the catalytic subunit of the polycomb repressive complex 2 (PRC2) which 
trimethylates lysine 27 on histone 3 (H3K27Me3), resulting in transcriptional silencing of numerous genes including tumor suppressors (12). EZH2 expression is increased in aggressive bladder cancer and promotes bladder cancer cell proliferation $(13,14)$.

Interestingly, work in drosophila, yeast, and ovarian clear cell carcinoma has revealed a functional antagonism between ARID1A and EZH2, and that mutations in ARID1A sensitize cells to EZH2 pharmacologic inhibition with the small molecule inhibitor GSK-126 both in vitro and in vivo (1517). We hypothesized that bladder cancer cells with ARID1A mutations would show sensitivity to EZH2 inhibition which could be utilized as a therapeutic target in patients with ARID1A-deficient bladder cancer.

Herein, we show that ARID1A-deficient BLCa is particularly sensitive to inhibition of EZH2 with the small molecule inhibitor GSK-126. Specifically, EZH2 inhibitor-treated ARID1A mutant and/or knockdown cells show significantly reduced cancer cell viability, colony formation, and xenograft growth relative to ARID1A-wild type cells. These data support the rationale for repurposing inhibitors of EZH2 to treat patients harboring advanced bladder cancers with ARID1A mutations. 


\section{Materials and Methods}

\section{Cell lines and reagents}

HEK293T (ATCC) and all bladder cancer cell lines HT1197 (ATCC, Manassas, VA, USA), HT1376 (ATCC), T24 (ATCC), 5637 (ATCC), RT112, VM-CUB1 (DSMZ, Braunschweig, Germany) were grown in Dulbecco's 90\% Dulbecco's MEM (4.5 g/L glucose) with penicillinstreptomycin $(100 \mathrm{U} / \mathrm{ml})$ and 10\% fetal bovine serum (Sigma-Aldrich, St Louis, MO, USA) in $5 \% \mathrm{CO}_{2}$ cell culture incubator. GSK-126 was obtained from Med Chem Express (catalog \# HY$13470)$.

\section{Human bladder cancer lysate preparation}

With IRB approval, and after pathologic stage and grade determination, protein lysates were prepared from human bladder cancer samples and surrounding normal mucosa, using samples from radical cystectomy (as previously described in ref (18)).

\section{In silico data analysis}

Using cBioPortal, the mutation profiles of ARID1A and EZH2 in muscle-invasive and non-muscle invasive bladder cancer were obtained. cBioPortal provides user-friendly graphical interface to analyze whole exome sequencing datasets from The Cancer Genome Atlas Project and other published reports $(2,5,19,20)$. To study EZH2 gene expression profile in bladder invasive carcinoma patients, TCGA level 3 RNA-seq data (including "raw_read_count" and "scaled_estimate" for each sample) was downloaded for all primary tumor and normal samples using TCGA-Assembler (21). Transcript per million values for each gene was obtained by multiplying scaled estimate by $1,000,000$. Using patient ID from cBioPortal, primary tumors were categorized based on ARID1A mutation status. Boxplot was generated using R (https://cran.rproject.org/). 


\section{ARID1A shRNA}

High titer lentivirus was generated by transfecting HEK293FT cells with a mixture containing three plasmids and 25-kDa linear polyethyleneimine (PEI) (Polysciences, Inc. PA, USA, 239662). In brief, $2.5 \mu \mathrm{g}$ pMD2.G (Addgene, MA, USA, 12259), $6.5 \mu \mathrm{g}$ psPAX2 (Addgene, 12260) and 3 $\mu \mathrm{g}$ pLKO.1-shARID1A (MISSION shRNA (Sigma-Aldrich), (TRCN0000059090 or TRCN0000059089) were diluted in $1.5 \mathrm{ml}$ Opti-MEM (reduced serum) (ThermoFisher, 11058021) medium and incubated for five minutes. Afterwards, $36 \mu 1$ of PEI was added to the plasmid mixture and incubated for $20 \mathrm{~min}$ at room temperature. The plasmid/PEI mixture was added to the HEK293FT cells (70-80\% confluence) grown in a T-75 flask and incubated in 5\% $\mathrm{CO} 2$ at $37^{\circ} \mathrm{C}$ overnight. The original medium was replaced with fresh medium [DMEM, 1x MEM Non-essential amino acid (ThermoFisher, 11140050) and Fetal Bovine Serum (ThermoFisher, 10082147) $18-20 \mathrm{~h}$ after transfection. The supernatant containing the first batch of the lentivirus was collected $24 \mathrm{~h}$ after replacement of the medium. This step was repeated and the second batch of lentivirus was collected after $48 \mathrm{~h}$. The two batches of lentivirus were combined and filtered through a $0.45 \mu \mathrm{m}$ filter. To concentrate the lentivirus, the filtrate was placed in a centrifuge tube containing Opti-prep ( 4 ml) (Sigma, D1556) at the bottom as the cushion and centrifuged at $50,000 \times \mathrm{g}$ for $2 \mathrm{~h}$ using an SW32Ti rotor (Beckman Coulter). After centrifugation, a layer containing the lentiviral particles located between the medium and Opti-prep was collected and placed in a 50-ml Falcon tube. Culture medium was added to the tube to increase the volume to 50 $\mathrm{ml}$. A second centrifugation was done at $5000 \times \mathrm{g}$ overnight at $4^{\circ} \mathrm{C}$. The pellet containing the lentiviral particles was re-suspended in ice-cold PBS (pH 7.4) and stored as $10 \mu 1$ aliquots at $80^{\circ} \mathrm{C}$. These lentiviruses were used to infect ARID1A wild-type BLCa cell lines (T24, 5637 and RT112) which were then selected for stably transfected clones using puromycin (Gibco, 
ThermoFisher), at the concentration of $1-2 \mu \mathrm{g} / \mathrm{ml}$ for 2 weeks. Stable clones were selected and tested for ARID1A knockdown using the methods below.

\section{Immunoblot analyses}

Antibodies used are noted in Table 1 below. All antibodies were used at dilutions optimized in our laboratory. For immunoblot analysis, protein samples were separated on a sodium dodecyl sulfatepolyacrylamide gel electrophoresis and transferred onto Immobilon-P PVDF membrane (EMD Millipore, Billerica, MA, USA). The membrane was incubated for $1 \mathrm{~h}$ in blocking buffer (Trisbuffered saline, $0.1 \%$ Tween (TBS-T), 5\% nonfat dry milk), followed by incubation overnight at $4{ }^{\circ} \mathrm{C}$ with the primary antibody. After a wash with TBS-T, the blot was incubated with horseradish peroxidase-conjugated secondary antibody and signals were visualized by Luminata Crescendo chemiluminescence western blotting substrate as per the manufacturer's protocol (EMD Millipore).

Table 1: List of antibodies used in this study, Related to the Materials and methods.

\begin{tabular}{|l|l|l|l|l|}
\hline Antibody & Application & Dilution & Supplier & Cat. No. \\
\hline ARID1A & IB & IB, 1:1000 & Cell Signaling Technology, Danvers, & D2A8U \\
\hline EZH2 & IB & IB, 1:1000 & Cell Signaling Technology, Danvers, & $5246 \mathrm{~S}$ \\
\hline H3K27Me3 & IB & IB, 1:2000 & Cell Signaling Technology, Danvers, & 9733S \\
& & & MA & \\
\hline
\end{tabular}




\begin{tabular}{|l|l|l|l|l|}
\hline Histone H3 & IB & IB, 1:2000 & Cell Signaling Technology, Danvers, & 4499S \\
& & MA & HRP-60008 \\
\hline $\boldsymbol{\beta}$-Actin & IB & IB, 1:100000 & PTG lab, Rosemont, IL & \\
\hline
\end{tabular}

\section{Cell proliferation assays}

Cell proliferation was measured by luminescence. For this, either ARID1A-mutant bladder cancer cells - HT1197, HT1376 and VMCUB1, or wild-type cells - T24, RT112, and 5637 were used. These cells were treated with DMSO, 7.5 or $10 \mu M$ of GSK-126 for 8 days while replenishing with the fresh drug + growth medium every other day. Measurements were made according to manufacturer's instructions. Briefly, plates were removed from the incubator and allowed to equilibrate at room temperature for 30 minutes, and equal volume of CellTiter-Glo ${ }^{\circledR} 2.0$ reagent was added directly to the wells. Plates were incubated 2 minutes on a shaker to induce cell lysis and then allow the plates to incubate at room temperature for 10 minutes to stabilize the luminescent signal. Luminescence was measured on a Synergy HTX multi-mode reader (BioTek Instruments, Inc., Winooski, VT, USA).

\section{Colony formation assay}

Bladder cancer cells were seeded at 800 cells per well of 6-well plates (triplicate) and incubated at $37{ }^{\circ} \mathrm{C}$ with $5 \% \mathrm{CO}_{2}$ for $7-10$ days while treating with GSK-126 every other day. Here both untreated and DMSO treated cells served as controls. Colonies were fixed with $10 \%$ (v/v) ethanol for $30 \mathrm{~min}$ and stained with crystal violet (Sigma-Aldrich, St Louis, MO, USA) for 20 min. Then, the photographs of the colonies were taken using Amersham Imager 600RGB (GE Healthcare Life Sciences, Pittsburgh, PA, USA). Colony quantification was carried out using ImageQuant TL Colony v.8.1 software (GE Healthcare Life Sciences). 


\section{Xenografts tumor growth assay}

Animal experiments were approved by the Institutional Animal Care and Use Committee of UAB.

For tumor xenograft experiments, NU/J nu/nu mice aged 6-8 weeks ( $\mathrm{n}=5$ for each group) from Jackson Laboratories were injected subcutaneously into the right dorsal flanks with human bladder cancer cell lines harboring ARID1A wild-type alleles (RT112 and 5637), ARID1A mutant alleles (HT1376 and VMCUB-1), or stable ARID1A knockdown (RT112 and 5637) $\left(1-2 \times 10^{6}\right.$ cells in $50 \mu \mathrm{L}$ of incomplete media without FBS, and $50 \mu \mathrm{L}$ of Matrigel). After inoculation of the cells, tumor growth was measured with Vernier calipers and recorded on a weekly basis. Tumor volume was calculated with formula: $0.5 \times$ tumor length $\times$ tumor width $^{2}$. For all in vivo studies, GSK-126 was administered intraperitoneally at a dose of $100 \mathrm{mg} / \mathrm{kg}$ once daily. The final volume of drug/vehicle was $0.2 \mathrm{ml}$ per $20 \mathrm{~g}$ body weight in $20 \%$ captisol adjusted to $\mathrm{pH} 4-4.5$ with $1 \mathrm{~N}$ acetic acid. Treatment with GSK-126 for a duration of 21 days was started after the tumor volume reached to 150 to $200 \mathrm{~mm}^{3}$ in size. At the end of the treatment, tumors were excised, weighed, processed and stored for downstream molecular analysis. 


\section{Results}

\section{Dysregulation of ARID1A in bladder cancer}

We and others have shown that EZH2 is critical for tumor cell survival, tumor growth, and regulates tumor suppressor gene and microRNA expression in aggressive prostate, breast, bladder and other cancers $(8,9,22)$. Our in silico mutation analysis via cBioPortal (http://www.cbioportal.org/), using the Memorial Sloan Kettering Cancer Center bladder cancer sequencing dataset and the TCGA dataset, indicated that up to $28 \%$ of bladder cancers harbor nonsense or truncating mutations in ARID1A (Fig. 1A). Furthermore, using protein lysates from tumors isolated from patients, we found that EZH2 protein levels were dramatically increased in tumors compared to surrounding normal urothelial mucosa, while ARID1A showed the opposite expression pattern (Fig. 1B). Histone H3-trimethylated lysine 27 levels were increased in tumor samples as expected, which correlated with overexpression of EZH2 (Fig. 1B). Furthermore, ARID1A-mutated bladder cancers express high level of EZH2 (Fig. 1C).

Our analysis of COSMIC cell line dataset (Wellcome Trust Sanger Institute, UK) suggested that multiple bladder cell lines harbored mutations in ARID1A. Among them, HT1197 has non-sense and missense substitution, HT1376 has frameshift deletion and missense substitution, and VMCUB1 has a non-sense substitution mutation in ARID1A gene. Other bladder cancer cell lines (T24, RT-112 and 5637) do not harbor mutations in the ARID1A gene. These data have been independently confirmed by other groups (23). Importantly, these cell lines harboring truncating mutations in ARID1A show a dramatic decrease in levels of the ARID1A protein (Fig 2A). 


\section{ARID1A mutation is associated with sensitivity to EZH2 inhibitors}

GSK-126 is a specific small molecule inhibitor of EZH2. To investigate the effect of GSK-126 on cell proliferation in ARID1A mutated bladder cancer cells, we performed cell viability experiments in cell lines with and without mutations in ARID1A. GSK-126 treatment significantly decreased cell viability in ARID1A mutated bladder cancer cell lines HT1197, HT1376 and VMCUB1. However, no significant change in cell number was seen in ARID1A wild type cells (Fig.2 C, E). To confirm that the doses used were effective in inhibiting the histone methyltransferase activity of EZH2, we performed immunoblot analysis for the EZH2 substrate H3K27Me3. As expected, GSK-126 decreased H3K27Me3 in all bladder cancer cell lines (Fig. 2 B, D). Thus, at these concentrations, ARID1A mutant bladder cancer cell lines are sensitive to EZH2 inhibition with GSK-126, while ARID1A wild-type cell lines are resistant.

To investigate these findings in vivo, we performed mouse xenograft experiments with ARID1A mutant and wild-type bladder cancer cell lines. Indeed, while xenografts harboring ARID1A mutations were sensitive to systemic GSK-126 treatment, xenografts with wild-type ARID1A were resistant (Fig 3 A-D, supp Fig 1 A-C). To confirm that EZH2 was still pharmacologically active in the ARID1A wild-type xenografts, protein lysates were subjected to H3K27Me3 immunoblotting (Supp Fig 2 A-C)

\section{ARID1A knockdown induces GSK-126 sensitivity in ARID1A wild-type bladder cancer cells}

To investigate whether ARID1A deficiency is sufficient for EZH2 inhibitor sensitivity, we generated ARID1A-wildtype bladder cell lines harboring stable shRNA-mediated knockdown of ARID1A. Notably, these cell lines showed ARID1A protein levels that are comparable to ARID1A 
mutant cell lines (Fig 4A). While ARID1A knockdown did not affect colony formation or cell viability at baseline, ARID1A knockdown did result in increased sensitivity to GSK-126 as evidenced by decreased colony formation (Fig 4B), decreased viability (Fig 4C), and increased pharmacologic sensitivity (10-fold decrease in IC50) (Fig 4D). These ARID1A stable knockdown cells were then used to generate xenografts to test their sensitivity to EZH2 inhibition in vivo. While RT112 and 5637 ARID1A wild-type cells were previously resistant to GSK-126 treatment (Fig 3 C, D), ARID1A knockdown cells were quite sensitive and their growth was nearly completely inhibited (Fig 4 E, F). Taken together, it was confirmed that sensitivity of bladder cancer cells towards an EZH2 inhibitor is dependent on ARID1A deficiency. 


\section{Discussion}

Metastatic urothelial carcinoma is generally incurable with modest survival benefit provided by cisplatin-based first-line chemotherapy (median survival $\sim 15$ months) (24-31). Durable benefits with post-platinum PD-1/L1 inhibitors extend to a minority of patients $(\sim 20 \%)$ and the median survival is $<1$ year $(25,32-36)(37,38)$. Third-line salvage therapies provide incremental benefits with median overall survival of $\sim 1$ year and are not curative (e.g. Enfortumab Vedotin).

Moreover, the first targeted agent, erdafitinib, was recently shown to be active and approved to treat post-platinum patients with activating somatic FGFR2/3 mutations or fusions (39-43). Hence, new therapeutic approaches are critically needed to yield cures, which will only arrive with better understanding of mechanisms of resistance and therapeutically actionable targets. Given the heterogeneity of this malignancy with multiple genomic alterations, there remains a role for rational approaches targeting these subsets of patients.

Recent studies have revealed that ARID1A is frequently mutated across a wide variety of human cancers including bladder, gastric, pancreatic, and ovarian cancers, and also has bona fide tumor suppressor properties $(6,7)$. As ARID1A is the DNA-binding subunit of the large $1.15 \mathrm{MDa}$ SWI/SNF multi-subunit complex, its loss through non-sense and missense mutations is thought to result in complex disassembly. Interestingly, loss of just one allele of ARID1A results in embryonic lethality in mice (44). Clearly, the protein levels of ARID1A are important in development and disease.

While loss of tumor suppressors like ARID1A can be difficult to therapeutically target directly, oftentimes these losses result in therapeutic vulnerabilities that can be targeted through a synthetic lethality approach. Using various bladder cancer cell lines with ARID1A truncating 
mutations or shRNA-mediated depletion, our experiments reveal that EZH2 inhibition is synthetically lethal in bladder cancer cells with ARID1A deficiency. Notably, similar findings have been discovered in ovarian clear cell carcinoma (15). Other groups have independently investigated this relationship in bladder cancer cells and come to somewhat different conclusions (23). On careful review, the cells in these studies were only treated with EZH2 inhibitor for 2-3 days and showed no specific sensitivity for EZH2 inhibition, whereas we have found that at least 6-8 days of treatment is necessary to see maximal differences in viability. This alone could explain the differences between our results.

Pharmacologic inhibitors of EZH2 are currently being investigated in a large variety of tumor types including lymphoma, sarcomas, and advanced treatment resistant solid tumors (reviewed in ref (45)). B-cell lymphomas often harbor activating mutations in EZH2, and some sub-types of sarcomas harbor mutations in SWI-SNF subunits SMARCB1 or SMARCA4. In fact, the EZH2 inhibitor tazemetostat was approved by the FDA in 2020 for the treatment of advanced epithelioid sarcoma. Thus, there is strong rationale to re-purpose EZH2 inhibitors for the pharmacologic treatment of bladder cancer patients whose tumors harbor ARID1A-mutations and/or deficiency. Notably, there is currently an active phase I/II trial investigating combination pembrolizumab and the EZH2 inhibitor tazemetostat in molecularly unselected advanced urothelial carcinoma (clinicaltrials.gov NCT03854474). The results herein suggest that subgroup analyses of this and other trials should focus on patients with ARID1A-deficient tumors.

We are currently actively investigating the molecular mechanisms of how ARID1A loss leads to EZH2 inhibitor sensitivity in bladder cancer. In ovarian carcinoma with clear cell histology, ARID1A and EZH2 both compete (along with histone deacetylases) to modulate the transcriptional activity of the PIK3IP1 gene $(15,46)$. PIK3IP1 is a $47 \mathrm{kDa}$ transmembrane protein 
which has been shown to directly bind to and inhibit the catalytic subunits of Class I PI3K, including PIK3CA (aka p110 $)(47,48)$. Thus, these studies showed that ARID1A loss combined with EZH2 inhibition results in a de-silencing of PIK3IP1 which inhibits $\mathrm{PI} 3 \mathrm{~K} / \mathrm{AKT} / \mathrm{mTOR}$ signaling resulting in inhibition of cellular proliferation. The $\mathrm{PI} 3 \mathrm{~K} / \mathrm{AKT} / \mathrm{mTOR}$ pathway is important in bladder cancer tumorigenesis, as a significant proportion of these tumors harbor activating mutations in PIK3CA (2). We therefore hypothesize that ARID1A loss in bladder cancer may result in PI3K/AKT/mTOR pathway activation and dependence that can be indirectly targeted by EZH2 inhibitor-mediated upregulation of PIK3IP1. Further studies are underway to investigate this hypothesis. In summary, our studies suggest a rationale for treating ARID1A mutated bladder cancers by targeting EZH2 with specific small molecule inhibitors. 


\section{FIGURE LEGENDS}

Figure 1: Bladder cancers harbors frequent inactivating mutations in ARID1A, and show low levels of ARID1A protein, and elevated EZH2 protein. (A) Oncoplots showing prevalence of ARID1A and EZH2 mutations in muscle invasive and non-muscle invasive bladder carcinoma. Mutation profile from three independent genomic sequencing data gathered from cBioPortal.org show significant number of bladder cancers harbor truncating or missense mutations in ARID1A. (B) Immunoblot of invasive bladder tumor (T) and matched normal tissue $(\mathrm{N})$ (from cystectomy specimens) shows expression pattern of EZH2 and ARID1A. Histone H3 trimethyl lysine 27 (H3K7me3) level is increased in BLCA corresponding to overexpression of EZH2. (C) Boxwhisker plot showing expression level of EZH2 in TCGA bladder carcinoma tumors with wild type and mutant ARID1A.

Figure 2: Bladder cancer cell lines with ARID1A mutation are sensitive to EZH2 inhibition. (A) Immunoblot shows expression of ARID1A in different bladder cancer cell lines including ARID1A mutant (HT1197, HT1376, and VMCUB-1), and ARID1A wild type (T24, 5637, and RT112) cell lines. (B) Immunoblot analysis showing expression level of EZH2, Histone H3 trimethyl lysine 27 (H3K27me3) and total Histone 3 in ARID1A mutant bladder cancer cell lines (HT1197, HT1376 and VM-CUB1) after treatment with EZH2 small molecule inhibitor GSK126. (C) Cell proliferation assay indicated ARID1A mutant bladder cancer cells are sensitive to GSK126. (D) Immunoblot analysis as above in (B) in ARID1A wild type bladder cancer cell lines (T24, 5637 and RT-112) after GSK126 treatment. (E) Cell proliferation assay of ARID1A wild type bladder cancer cells showed no effect of GSK126 treatment. "ns" - non-significant.

Figure 3: Bladder cancer xenografts harboring ARID1A mutations are sensitive to EZH2 inhibition. (A-D) Plots of tumor volume at indicated days after mice inoculated with HT1376 (ARID1A mutant), VMCUB-1 (ARID1A mutant), RT-112 (ARID1A wild type), and 5637 (ARID1A wild type) cells respectively, treated with EZH2 inhibitor GSK126 (dashed line) or vehicle (solid line). "ns" - non-significant.

Figure 4: ARID1A knockdown sensitizes bladder cancer cells to EZH2 inhibition. (A) Immunoblots showing expression of ARID1A, EZH2 and tri-methylated H3K27 (H3K27me3) in ARID1A wild type BLCa cell lines after ARID1A stable knockdown (KD) with two separate shRNA sequences along with scrambled (scr) shRNA. (B) Colony formation assays of untreated, scramble shRNA, and ARID1A KD 5637 cells with DMSO or GSK126 treatment. (C) Plots demonstrate the cell proliferation in parental (scr) ARID1Awt and KD cells and (D) Plots show the dose-response viability curves of ARID1A wild type (T24, 5637 and RT112) bladder cancer cell lines with and without ARID1Akd treated with GSK126 for 144h. (E, F) Plots of tumor volume at indicated days after mice inoculated with RT-112 (ARID1A knockdown), and 5637 (ARID1A knockdown) cells respectively, treated with EZH2 inhibitor GSK126 (dashed line) or vehicle (solid line). Inset, photomicrographs of xenograft tumors. 


\section{References}

1. Siegel RL, Miller KD, Jemal A. Cancer statistics, 2018. CA Cancer J Clin. 2018;68(1):7-30.

2. Robertson AG, Kim J, Al-Ahmadie H, Bellmunt J, Guo G, Cherniack AD, et al. Comprehensive Molecular Characterization of Muscle-Invasive Bladder Cancer. Cell. 2017;171(3):540-56 e25. PMC5687509

3. Hurst CD, Knowles MA. Mutational landscape of non-muscle-invasive bladder cancer. Urol Oncol. 2018.

4. Hurst CD, Alder O, Platt FM, Droop A, Stead LF, Burns JE, et al. Genomic Subtypes of Non-invasive Bladder Cancer with Distinct Metabolic Profile and Female Gender Bias in KDM6A Mutation Frequency. Cancer cell. 2017;32(5):701-15.e7. PMC5774674

5. Pietzak EJ, Bagrodia A, Cha EK, Drill EN, lyer G, Isharwal S, et al. Next-generation Sequencing of Nonmuscle Invasive Bladder Cancer Reveals Potential Biomarkers and Rational Therapeutic Targets. Eur Urol. 2017;72(6):952-9. PMC6007852

6. Hodges C, Kirkland JG, Crabtree GR. The Many Roles of BAF (mSWI/SNF) and PBAF Complexes in Cancer. Cold Spring Harb Perspect Med. 2016;6(8). PMC4968166

7. Mathur R. ARID1A loss in cancer: Towards a mechanistic understanding. Pharmacol Ther. 2018;190:15-23.

8. Varambally S, Dhanasekaran SM, Zhou M, Barrette TR, Kumar-Sinha C, Sanda MG, et al. The polycomb group protein EZH2 is involved in progression of prostate cancer. Nature. 2002;419(6907):6249.

9. Kleer CG, Cao Q, Varambally S, Shen R, Ota I, Tomlins SA, et al. EZH2 is a marker of aggressive breast cancer and promotes neoplastic transformation of breast epithelial cells. Proc Natl Acad Sci U S A. 2003;100(20):11606-11. PMC208805

10. Bracken AP, Pasini D, Capra M, Prosperini E, Colli E, Helin K. EZH2 is downstream of the pRB-E2F pathway, essential for proliferation and amplified in cancer. EMBO J. 2003;22(20):5323-35. PMC213796

11. Kim KH, Roberts CW. Targeting EZH2 in cancer. Nat Med. 2016;22(2):128-34. PMC4918227

12. Margueron R, Reinberg D. The Polycomb complex PRC2 and its mark in life. Nature. 2011;469(7330):343-9. PMC3760771

13. Weikert S, Christoph F, Kollermann J, Muller M, Schrader M, Miller K, et al. Expression levels of the EZH2 polycomb transcriptional repressor correlate with aggressiveness and invasive potential of bladder carcinomas. Int J Mol Med. 2005;16(2):349-53.

14. Zhang YB, Niu HT, Chang JW, Dong GL, Ma XB. EZH2 silencing by RNA interference inhibits proliferation in bladder cancer cell lines. Eur J Cancer Care (Engl). 2011;20(1):106-12.

15. Bitler BG, Aird KM, Garipov A, Li H, Amatangelo M, Kossenkov AV, et al. Synthetic lethality by targeting EZH2 methyltransferase activity in ARID1A-mutated cancers. Nat Med. 2015;21(3):231-8. PMC4352133

16. Kim KH, Kim W, Howard TP, Vazquez F, Tsherniak A, Wu JN, et al. SWI/SNF-mutant cancers depend on catalytic and non-catalytic activity of EZH2. Nat Med. 2015;21(12):1491-6. PMC4886303

17. Wu S, Fatkhutdinov N, Fukumoto T, Bitler BG, Park PH, Kossenkov AV, et al. SWI/SNF catalytic subunits' switch drives resistance to EZH2 inhibitors in ARID1A-mutated cells. Nat Commun. 2018;9(1):4116.

18. Chandrashekar DS, Chakravarthi B, Robinson AD, Anderson JC, Agarwal S, Balasubramanya SAH, et al. Therapeutically actionable PAK4 is amplified, overexpressed, and involved in bladder cancer progression. Oncogene. 2020;39(20):4077-91. 
19. Cerami E, Gao J, Dogrusoz U, Gross BE, Sumer SO, Aksoy BA, et al. The cBio cancer genomics portal: an open platform for exploring multidimensional cancer genomics data. Cancer Discov. 2012;2(5):401-4. PMC3956037

20. Kim PH, Cha EK, Sfakianos JP, Iyer G, Zabor EC, Scott SN, et al. Genomic predictors of survival in patients with high-grade urothelial carcinoma of the bladder. Eur Urol. 2015;67(2):198-201. PMC4312739 21. Zhu Y, Qiu P, Ji Y. TCGA-assembler: open-source software for retrieving and processing TCGA data. Nat Methods. 2014;11(6):599-600. PMC4387197

22. Bachmann IM, Halvorsen OJ, Collett K, Stefansson IM, Straume O, Haukaas SA, et al. EZH2 expression is associated with high proliferation rate and aggressive tumor subgroups in cutaneous melanoma and cancers of the endometrium, prostate, and breast. J Clin Oncol. 2006;24(2):268-73.

23. Garczyk S, Schneider U, Lurje I, Becker K, Vogeli TA, Gaisa NT, et al. ARID1A-deficiency in urothelial bladder cancer: No predictive biomarker for EZH2-inhibitor treatment response? PLoS One. 2018;13(8):e0202965. PMC6107234

24. von der Maase H, Sengelov L, Roberts JT, Ricci S, Dogliotti L, Oliver T, et al. Long-term survival results of a randomized trial comparing gemcitabine plus cisplatin, with methotrexate, vinblastine, doxorubicin, plus cisplatin in patients with bladder cancer. J Clin Oncol. 2005;23(21):4602-8.

25. Sharma P, Retz M, Siefker-Radtke A, Baron A, Necchi A, Bedke J, et al. Nivolumab in metastatic urothelial carcinoma after platinum therapy (CheckMate 275): a multicentre, single-arm, phase 2 trial. Lancet Oncol. 2017.

26. Powles T, O'Donnell PH, Massard C, Arkenau HT, Friedlander TW, Hoimes CJ, et al. Efficacy and Safety of Durvalumab in Locally Advanced or Metastatic Urothelial Carcinoma: Updated Results From a Phase 1/2 Open-label Study. JAMA Oncol. 2017:e172411.

27. Bellmunt J, de Wit R, Vaughn DJ, Fradet Y, Lee JL, Fong L, et al. Pembrolizumab as Second-Line Therapy for Advanced Urothelial Carcinoma. N Engl J Med. 2017;376(11):1015-26. PMC5635424

28. Bellmunt J, von der Maase H, Mead GM, Skoneczna I, De Santis M, Daugaard G, et al. Randomized phase III study comparing paclitaxel/cisplatin/gemcitabine and gemcitabine/cisplatin in patients with locally advanced or metastatic urothelial cancer without prior systemic therapy: EORTC Intergroup Study 30987. J Clin Oncol. 2012;30(10):1107-13. 3341152

29. De Santis M, Bellmunt J, Mead G, Kerst JM, Leahy M, Maroto P, et al. Randomized phase II/III trial assessing gemcitabine/carboplatin and methotrexate/carboplatin/vinblastine in patients with advanced urothelial cancer who are unfit for cisplatin-based chemotherapy: EORTC study 30986. J Clin Oncol. 2012;30(2):191-9. 3255563

30. Galsky MD, Hahn NM, Rosenberg J, Sonpavde G, Hutson T, Oh WK, et al. Treatment of patients with metastatic urothelial cancer "unfit" for Cisplatin-based chemotherapy. J Clin Oncol. 2011;29(17):2432-8.

31. Galsky MD, Chen GJ, Oh WK, Bellmunt J, Roth BJ, Petrioli R, et al. Comparative effectiveness of cisplatin-based and carboplatin-based chemotherapy for treatment of advanced urothelial carcinoma. Ann Oncol. 2012;23(2):406-10.

32. Sonpavde G. PD-1 and PD-L1 Inhibitors as Salvage Therapy for Urothelial Carcinoma. N Engl J Med. 2017;376(11):1073-4.

33. Rosenberg JE, Hoffman-Censits J, Powles T, van der Heijden MS, Balar AV, Necchi A, et al. Atezolizumab in patients with locally advanced and metastatic urothelial carcinoma who have progressed following treatment with platinum-based chemotherapy: a single-arm, multicentre, phase 2 trial. Lancet. 2016;387(10031):1909-20.

34. Massard C, Gordon MS, Sharma S, Rafii S, Wainberg ZA, Luke J, et al. Safety and Efficacy of Durvalumab (MEDI4736), an Anti-Programmed Cell Death Ligand-1 Immune Checkpoint Inhibitor, in Patients With Advanced Urothelial Bladder Cancer. J Clin Oncol. 2016;34(26):3119-25. 
35. Bellmunt J, de Wit R, Vaughn DJ, Fradet $Y$, Lee JL, Fong L, et al. Pembrolizumab as Second-Line Therapy for Advanced Urothelial Carcinoma. N Engl J Med. 2017.

36. Patel MR, Ellerton J, Infante JR, Agrawal M, Gordon M, Aljumaily R, et al. Avelumab in metastatic urothelial carcinoma after platinum failure (JAVELIN Solid Tumor): pooled results from two expansion cohorts of an open-label, phase 1 trial. Lancet Oncol. 2018;19(1):51-64.

37. Balar AV, Galsky MD, Rosenberg JE, Powles T, Petrylak DP, Bellmunt J, et al. Atezolizumab as firstline treatment in cisplatin-ineligible patients with locally advanced and metastatic urothelial carcinoma: a single-arm, multicentre, phase 2 trial. Lancet. 2017;389(10064):67-76.

38. Powles T PS, Voog E, et al. Maintenance avelumab + best supportive care (BSC) versus BSC alone after platinum-based first-line (1L) chemotherapy in advanced urothelial carcinoma (UC): JAVELIN Bladder 100 phase III interim analysis. 101200/JCO20203818_supplLBA1.

39. Loriot Y, Necchi A, Park SH, Garcia-Donas J, Huddart R, Burgess E, et al. Erdafitinib in Locally Advanced or Metastatic Urothelial Carcinoma. N Engl J Med. 2019;381(4):338-48.

40. Rosenberg JE, O'Donnell PH, Balar AV, McGregor BA, Heath El, Yu EY, et al. Pivotal Trial of Enfortumab Vedotin in Urothelial Carcinoma After Platinum and Anti-Programmed Death 1/Programmed Death Ligand 1 Therapy. J Clin Oncol. 2019;37(29):2592-600. PMC6784850

41. Petrylak DP PR, Zhang J, et al. A Phase I Study of Enfortumab Vedotin: Updated Analysis of Patients with Metastatic Urothelial Cancer. J Clin Oncol 35, 2017 (suppl; abstr 106).

42. Tagawa ST, Faltas BM, Lam ET, Saylor PJ, Bardia A, Hajdenberg J, et al. Sacituzumab govitecan (IMMU-132) in patients with previously treated metastatic urothelial cancer (mUC): Results from a phase I/II study. Journal of Clinical Oncology. 2019;37(7_suppl):354-.

43. Siefker-Radtke AO, Necchi A, Park SH, GarcÃa-Donas Js, Huddart RA, Burgess EF, et al. First results from the primary analysis population of the phase 2 study of erdafitinib (ERDA; JNJ-42756493) in patients (pts) with metastatic or unresectable urothelial carcinoma (mUC) and FGFR alterations (FGFRalt). Journal of Clinical Oncology. 2018;36(15_suppl):4503-.

44. Gao X, Tate P, Hu P, Tjian R, Skarnes WC, Wang Z. ES cell pluripotency and germ-layer formation require the SWI/SNF chromatin remodeling component BAF250a. Proc Natl Acad Sci U S A. 2008;105(18):6656-61. PMC2373334

45. Eich ML, Athar M, Ferguson JE, Varambally S. EZH2-targeted therapies in cancer: hype or a reality. Cancer Res. 2020.

46. Fukumoto T, Park PH, Wu S, Fatkhutdinov N, Karakashev S, Nacarelli T, et al. Repurposing PanHDAC Inhibitors for ARID1A-Mutated Ovarian Cancer. Cell Rep. 2018;22(13):3393-400. PMC5903572

47. He X, Zhu Z, Johnson C, Stoops J, Eaker AE, Bowen W, et al. PIK3IP1, a negative regulator of PI3K, suppresses the development of hepatocellular carcinoma. Cancer Res. 2008;68(14):5591-8. PMC2753226

48. Zhu Z, He X, Johnson C, Stoops J, Eaker AE, Stoffer DS, et al. PI3K is negatively regulated by PIK3IP1, a novel p110 interacting protein. Biochem Biophys Res Commun. 2007;358(1):66-72. PMC1978172 


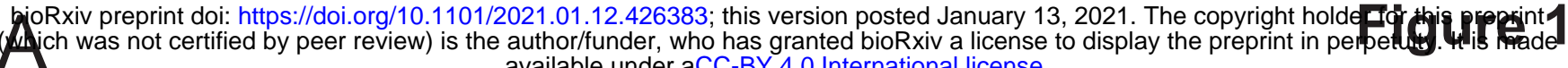

Muscle Invasive Bladder Cancer (MSKCC, Eur Urol 2014)

ARID1A $28 \%$ mmmmmmmmmmmm

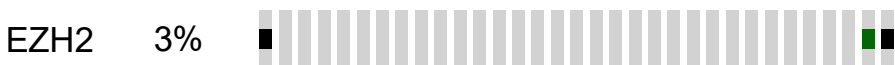

Muscle Invasive Bladder Cancer (TCGA, Cell 2017)

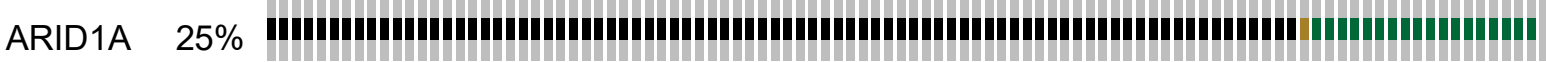

EZH2 $1.5 \%$

Non-muscle Invasive Bladder Cancer (MSK Eur Urol 2017)

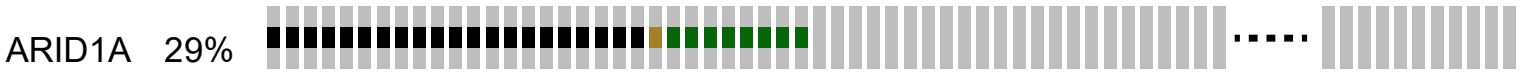

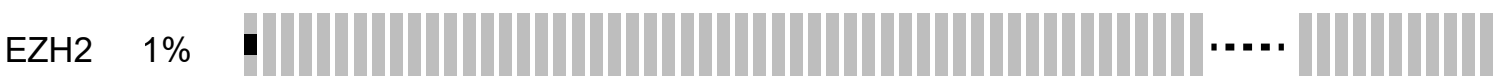

Inframe Mutation (unknown significance)

Truncating Mutation (putative driver)
Missense Mutation (unknown significance)

No alterations
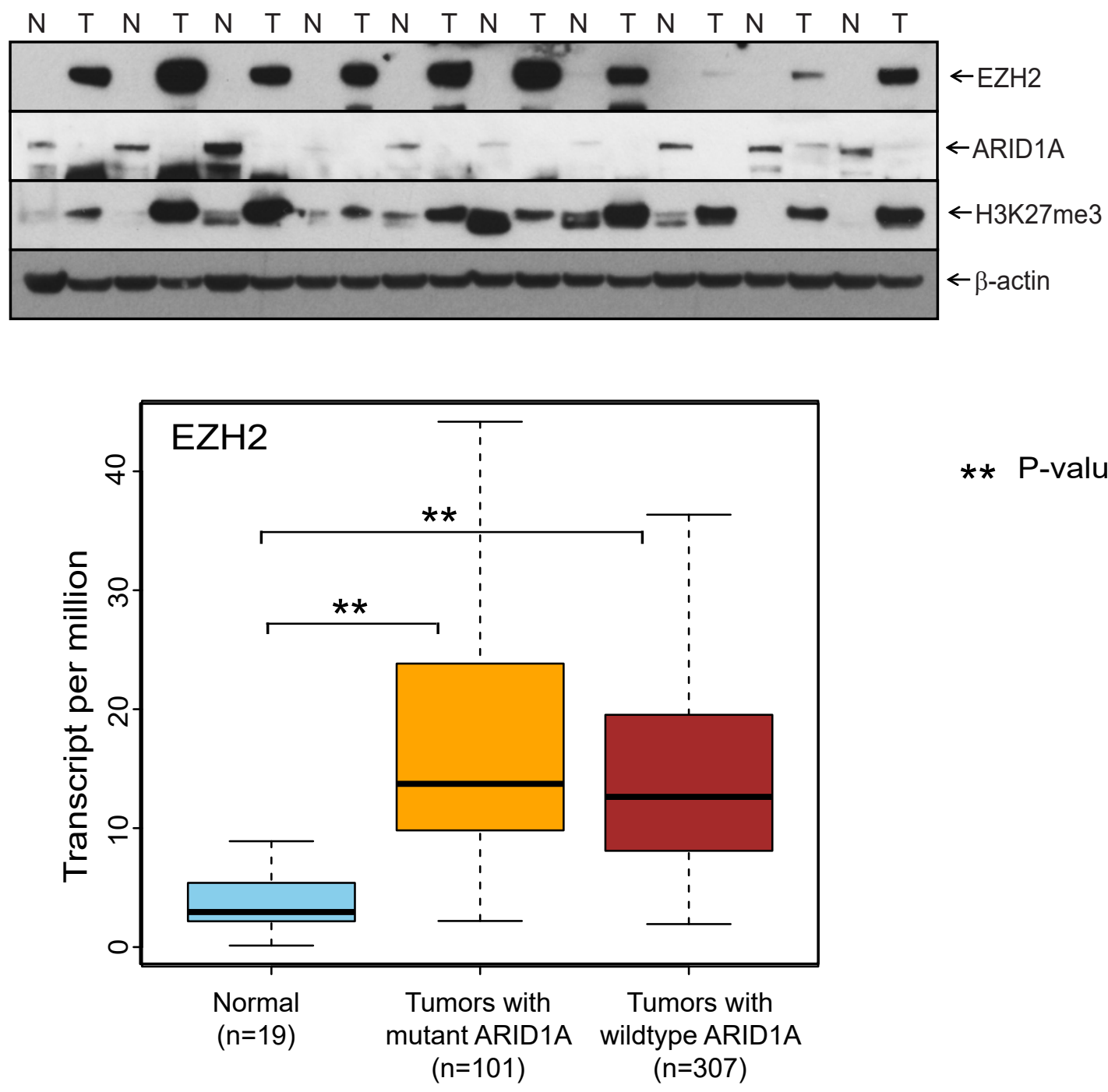

** P-value $<0.0005$ 
bioRxiv preprint doi: https://doi.org/10.1101/2021.01.12.426383; this version posted January 13,2021 . The copyright holder for this preprint (which was not certified by per review) is the author/funder, who has granted bioRxiv a license to display the preprint in perpetuity. It is made

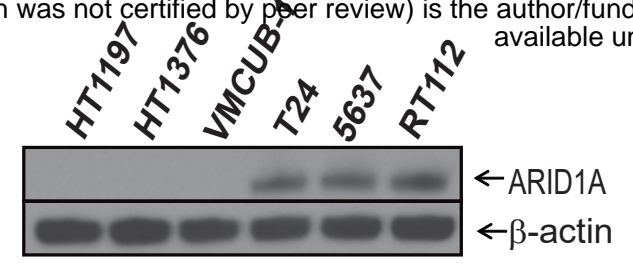

Figure 2
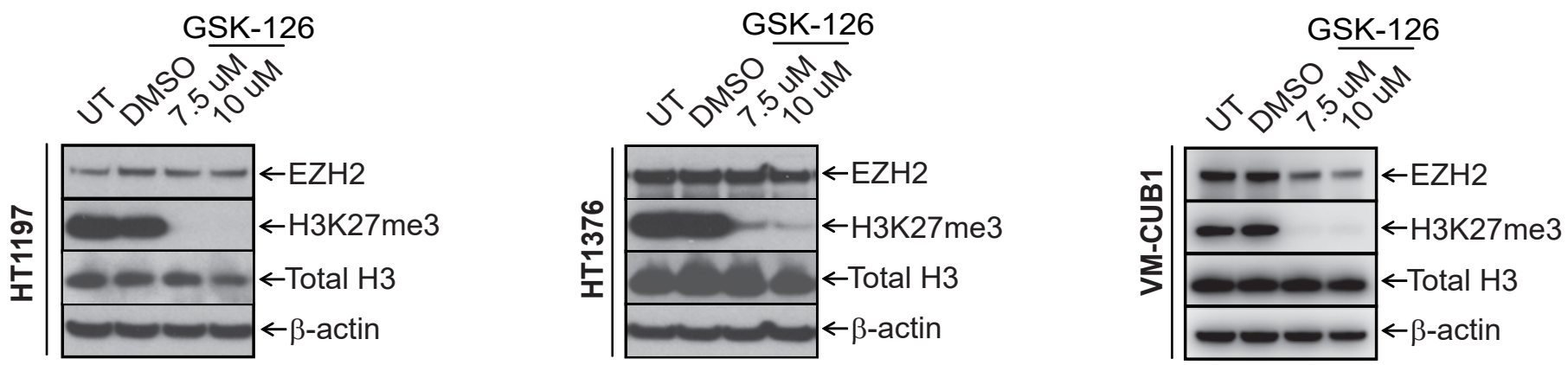

c
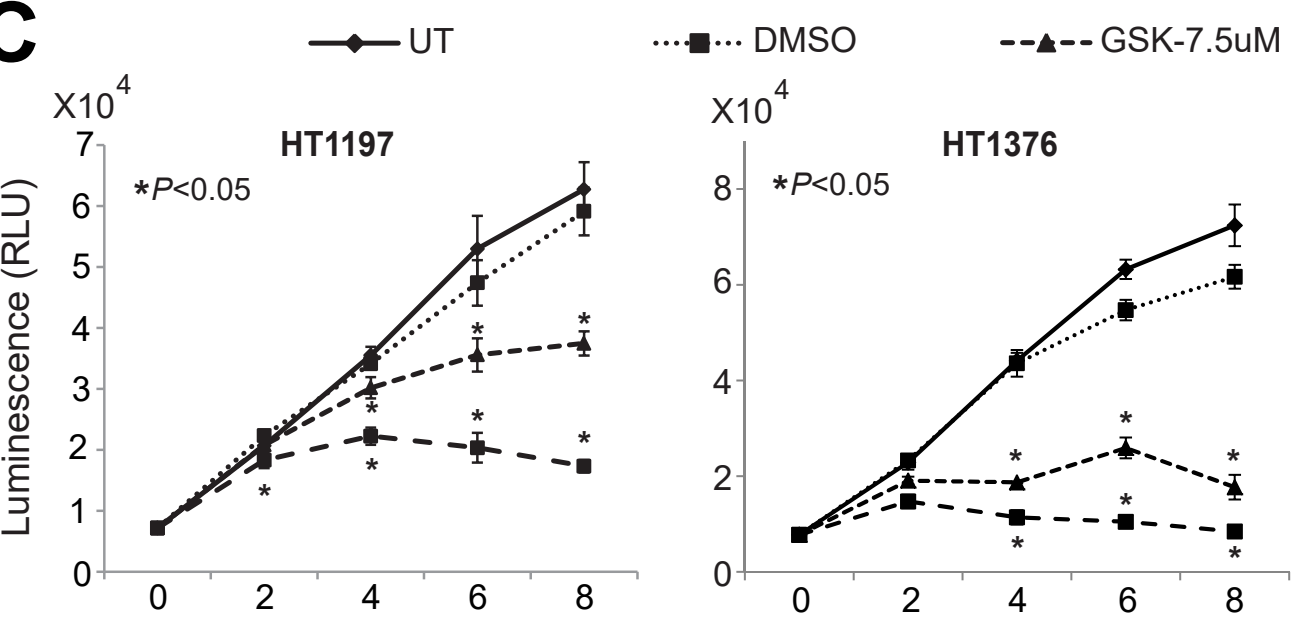

D
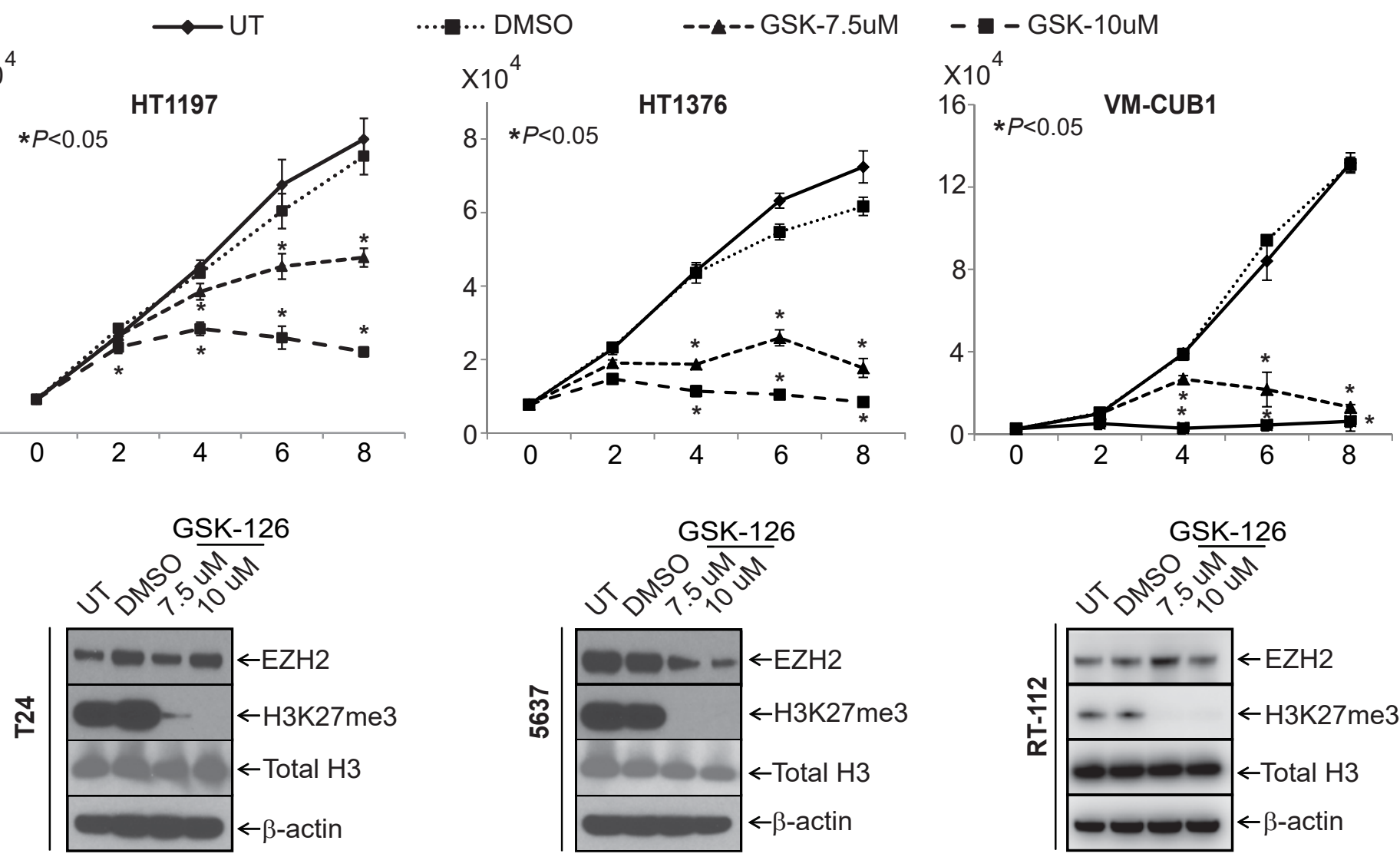

Days
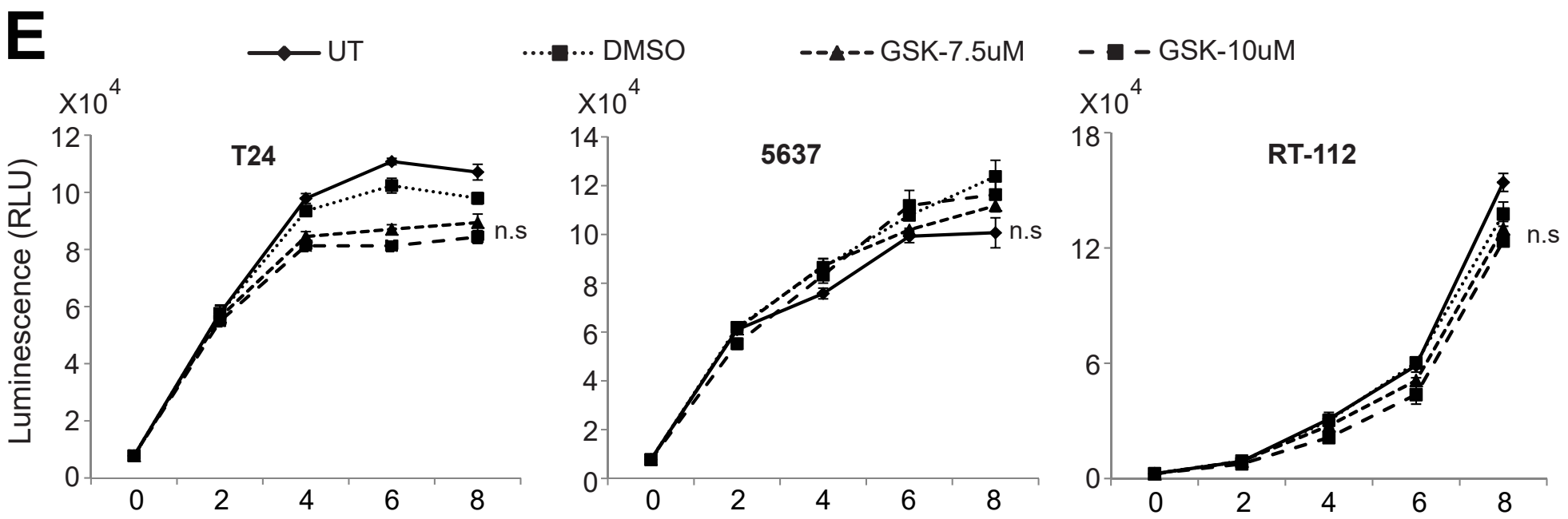

Days 
A

HT1376 (ARID1Amut)

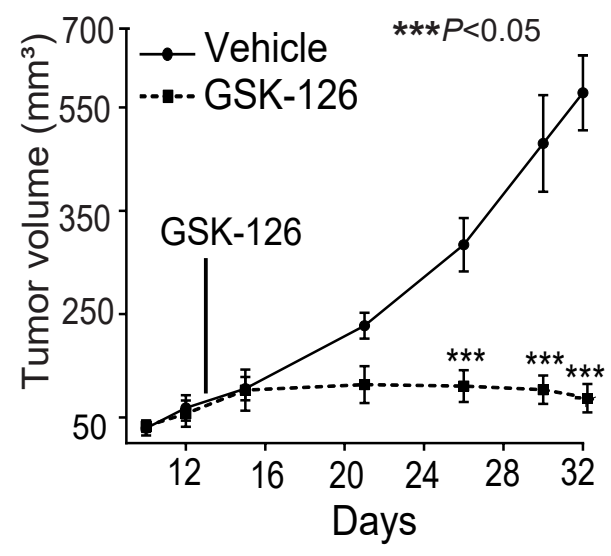

C

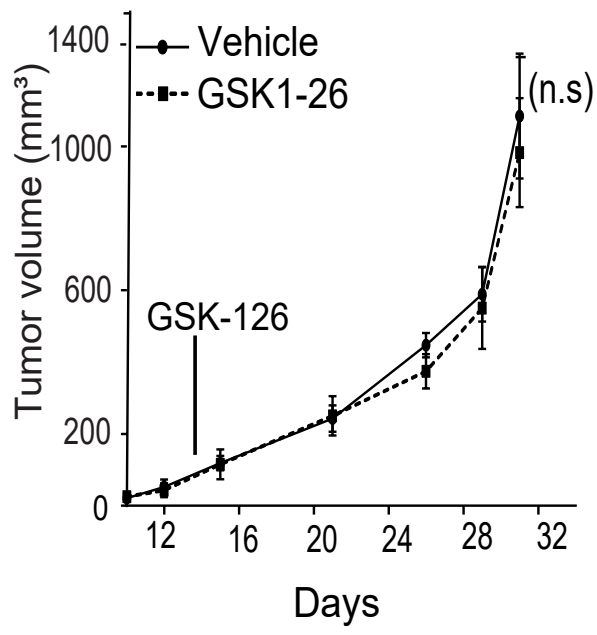

B

VMCUB-1 (ARID1Amut)

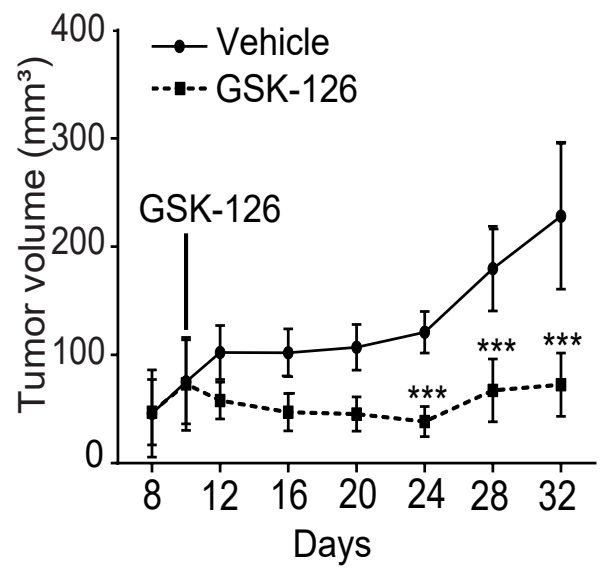

D $5637($ ARID1Awt)

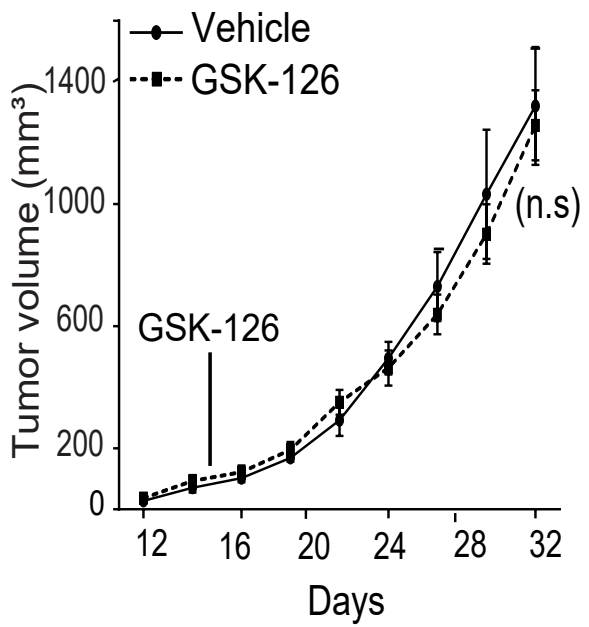


A

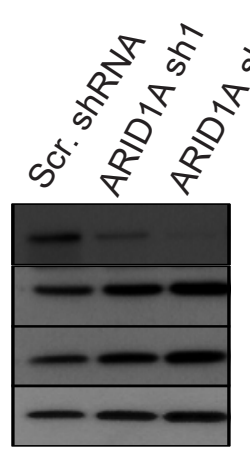

RT112

\section{is}

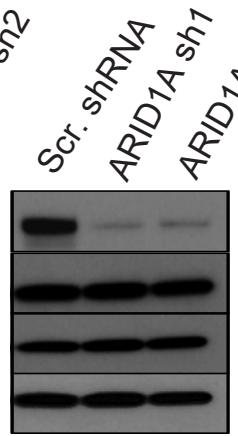

T24

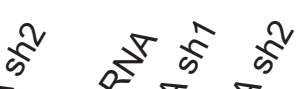

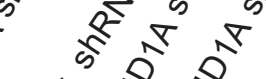

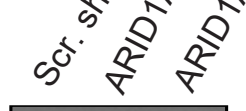

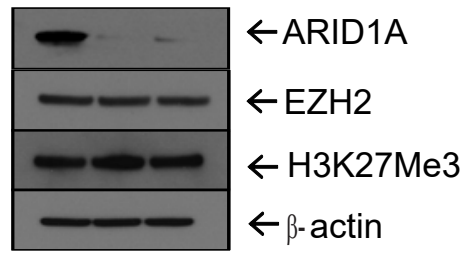

GSK126

(7.5 uM)

\section{ARID1A shRNA Scr.shRNA 5637 WT}

DMSO

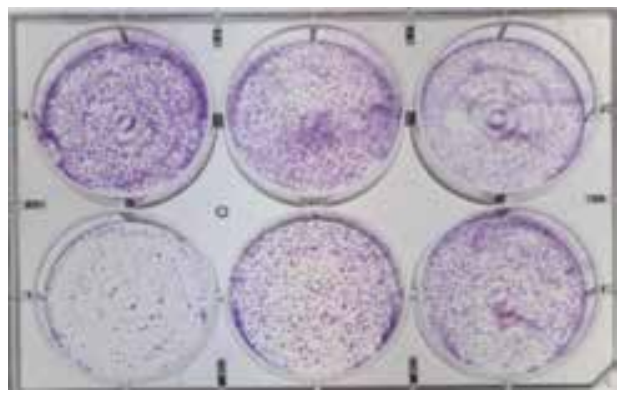

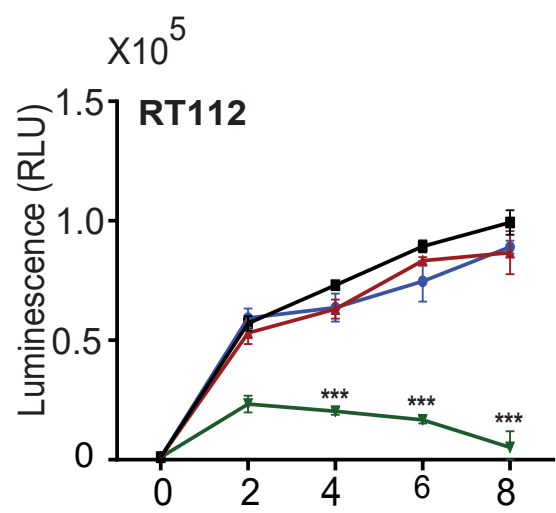
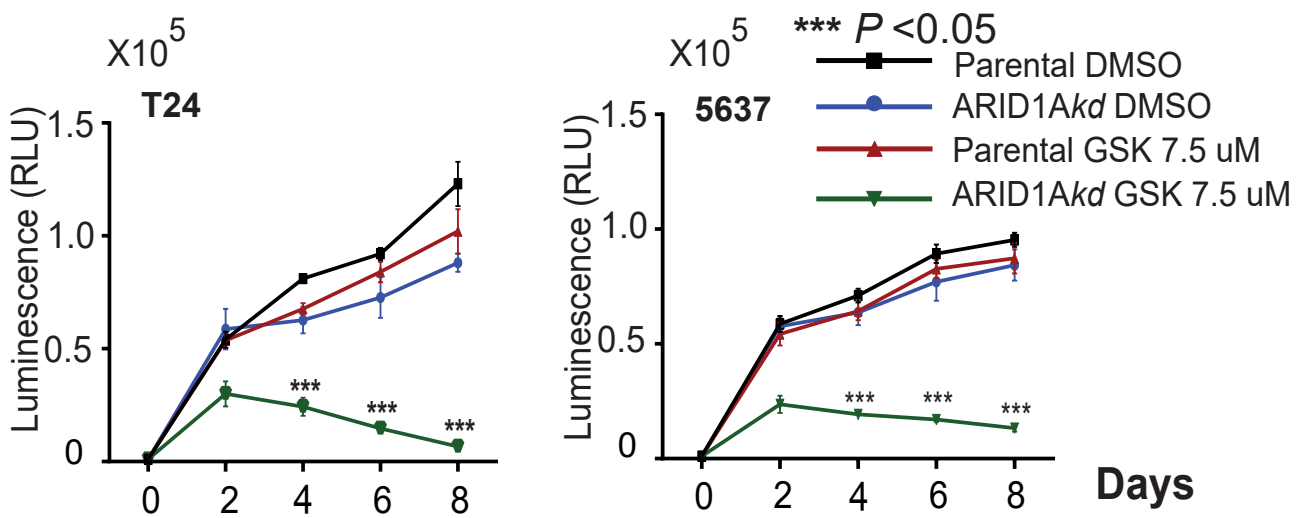

$D$

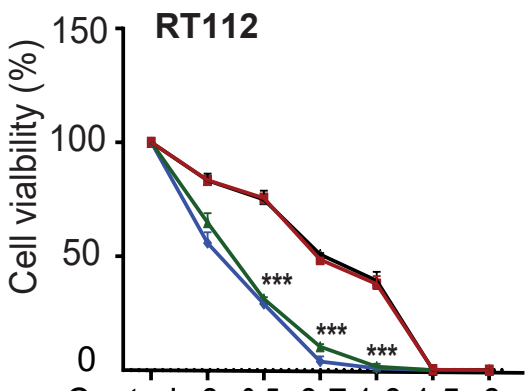

Control $0 \quad 0.5 \quad 0.71 .01 .52$ GSK-126 $\log _{10}(\mathrm{uM})$

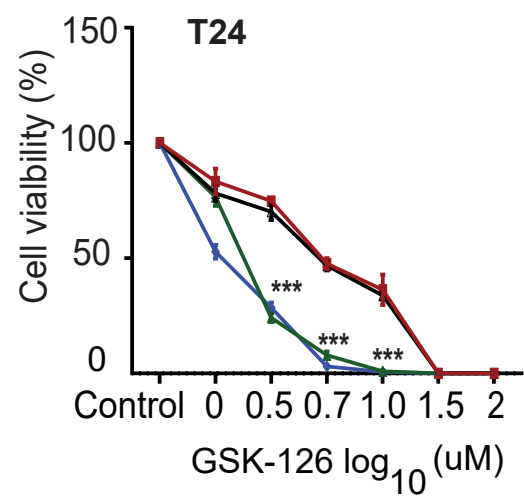

F

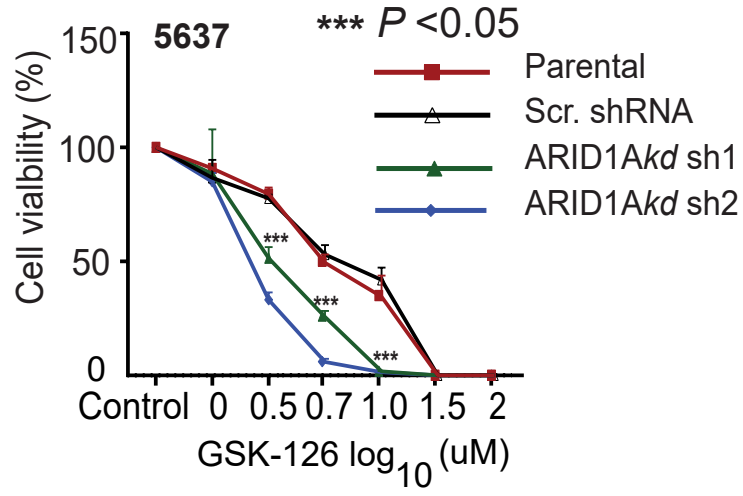

5637 (ARID1A kd)

है

RT112 (ARID1A kd)
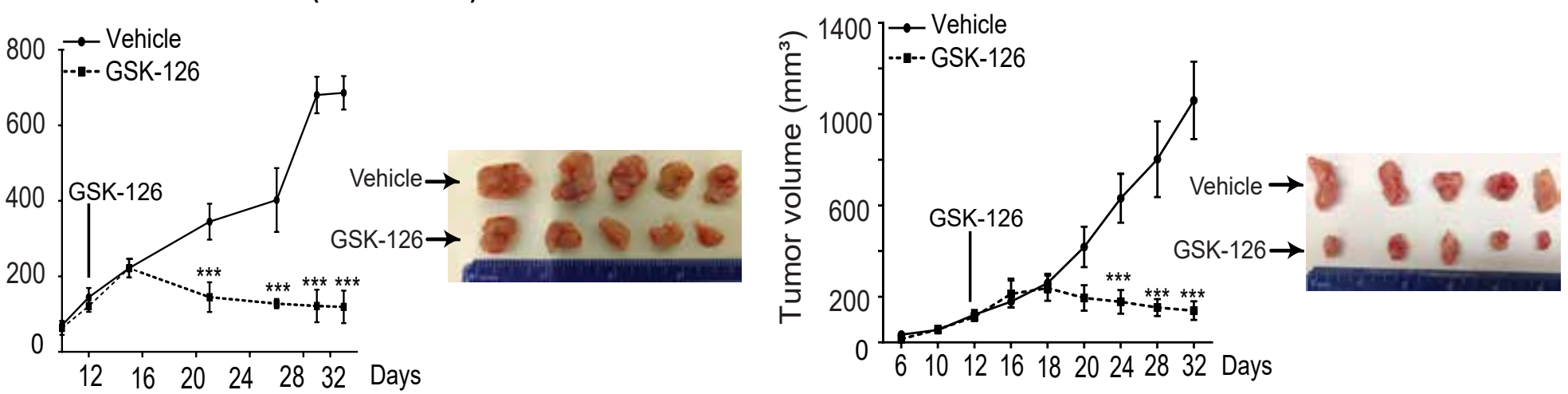

$* * * P<0.05$ 